\title{
Effect of nanorope waviness on the effective moduli of nanotube sheets
}

\author{
L. Berhan and Y. B. Yi \\ Department of Mechanical Engineering, University of Michigan, Ann Arbor, Michigan
}

\begin{abstract}
A. M. Sastry ${ }^{a)}$
Department of Mechanical Engineering and Department of Biomedical Engineering, University of Michigan, Ann Arbor, Michigan
\end{abstract}

(Received 8 September 2003; accepted 27 January 2004)

\begin{abstract}
Using a micromechanics approach, we recently investigated the theoretical limits on achievable moduli in nanotube mats by stiffening of bonds. However, the waviness intrinsic to many manufacturing processes also clearly plays an important role in stiffness of these materials. To study the effect of waviness on mechanical properties, we modeled fiber segments as sinusoids, generated networks comprised of these fibers, and performed simulations of deformations of the networks. In contradiction of classical work by Kallmes and Corte [Tappi J. 43, 737 (1960)], we found the number of fiber crossings in these networks to be independent of fiber waviness, leading to identification of the number of fiber crossings as a necessary and sufficient parameter to specify network geometry, for either wavy or straight fibers. Our mechanical modeling results suggest that reducing the waviness of nanotube ropes would significantly improve Young's moduli in these materials. However, reduction of waviness would not produce the improvements achievable with higher bond density; for random sheets, assuring connections among all intersecting ropes appears to be the most direct route toward improving the overall sheet properties. There remains a persistent discrepancy between statistically predicted bond densities and physical bond densities, based on moduli of these materials. (c) 2004 American Institute of Physics. [DOI: 10.1063/1.1687989]
\end{abstract}

\section{INTRODUCTION}

Improvements in nanotube (NT) sheet properties have been sought through stiffening of inter-rope/intertube connections $^{1-3}$ and alignment of fibers and ropes. ${ }^{4-7}$ Using a micromechanics approach, we have recently investigated the theoretical limits on achievable moduli by stiffening of bonds. ${ }^{1}$ However, the waviness intrinsic to many manufacturing processes also clearly plays an important role in material stiffness. For example, Fisher et al. ${ }^{8}$ studied NT curvature in NT reinforced polymer composites, and demonstrated that effective moduli are significantly reduced with increased waviness of embedded NTs. Here, we further develop a general methodology for linking NT, nanorope, and nanotube mat properties.

Single-walled carbon nanotubes are synthesized in closepacked bundles or ropes due to van der Waals forces. ${ }^{9,10} \mathrm{NT}$ ropes have very low bending rigidity, ${ }^{10}$ and thus readily form into porous composites of entangled, randomly oriented ropes and nanoscale impurities. Some of these impurities can be removed by acid treatment; dispersion of the NTs is commonly achieved using a surfactant. Filtration of the resulting suspension produces a porous NT mat, or "Bucky paper,"11 comprised of nanoropes with intrinsic curvature.

Previously, in investigating the effect of bond properties on modulus, we modeled the NT ropes as straight beams. ${ }^{1}$ We emphasize that our assumption of rigid bonds at fiber crossings is clearly an idealization. Although several ap-

\footnotetext{
a) Author to whom correspondence should be addressed; electronic mail: amsastry@umich.edu
}

proaches have been devised recently to create higher densities of bonds in NT sheets, ${ }^{1}$ our method does offer a means of determining the relative effects of improving bond density with fiber straightening on sheet properties. We thus set out the following three objectives:

(1) to develop a model for single NT rope curvature encompassing realistic arrangements of NTs in NT mats,

(2) to determine the effect of curvature on bond density and other geometric descriptors of NT mats, and

(3) to determine the effect of curvature on segment response, and overall mat properties.

We use both two-beam and network assemblies to study the effects of curvature, and also comment on the relative importance of bonds, segment curvatures, and other geometric features in NT mat properties.

\section{MODEL DEVELOPMENT}

Scanning electron microscope (SEM) images of NT sheets reveal some straightening of the ropes along torn edges. ${ }^{12}$ Thus, images analyzed here were taken in the surface planes of the sheets, wherein various curvatures of ropes and segments were observed [Fig. 1(a)]. Several nanoropes in each image were selected for analysis, to provide input to our geometric model (Fig. 2). SEM images of each of the two types of sheets considered [comprised of NTs manufactured using laser ablation and high pressure carbon monoxide (HiPCO) synthesis, respectively, and provided by the NanoTech Institute of the University of Texas at Dallas] were analyzed; five surface, sinusoidal ropes were selected from each image. 


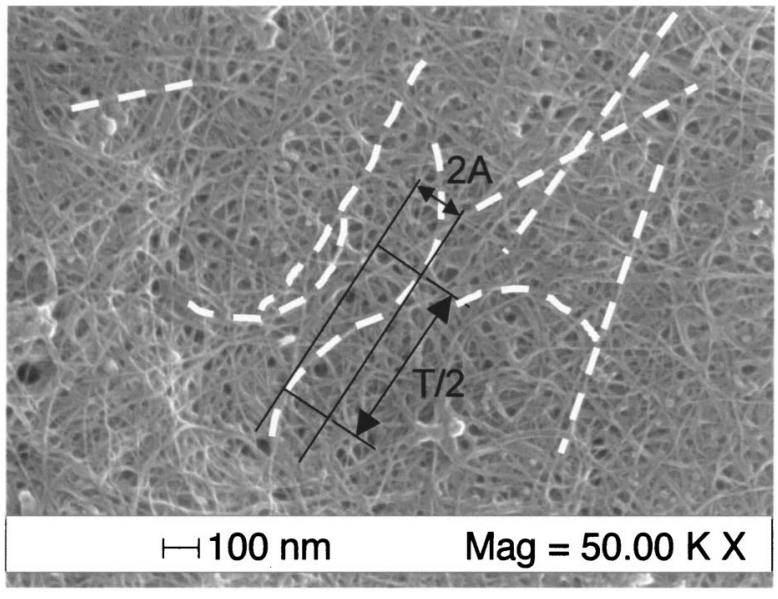

(a)

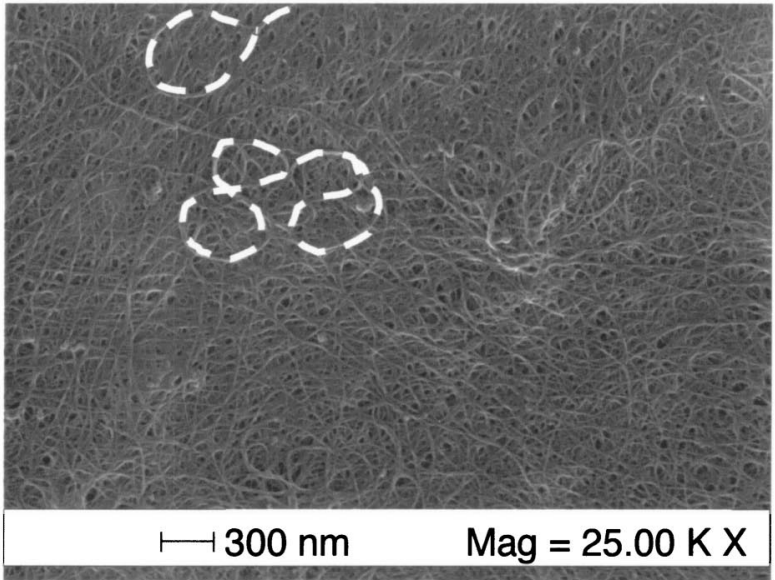

(b)

FIG. 1. SEM images of NT sheets showing (a) straight and sinusoidal segments and (b) closed loops.

Period $T$ and amplitude $A$ were first determined for each nanorope, represented by the sinusoid

$$
y=A \sin (\omega x+\alpha) .
$$

Period $T$ can be written as $2 \pi / \omega$, where $\omega$ is the angular frequency. The running length $L$ of a fiber from $x=0$ to $x$ $=a$, is given by

$$
L=\int_{0}^{a} \sqrt{1+A^{2} \omega^{2} \cos ^{2}(\omega x+\alpha)} d x .
$$

The coordinates of four points on each fiber, along with the orientation angle $\theta$ (as shown in Fig. 2), were recorded. ${ }^{13}$ These were rewritten in terms of $x^{\prime}$ and $y^{\prime}$, where the $x^{\prime}-y^{\prime}$ coordinate system is rotated about the $x-y$ coordinate system by $\theta$. After translation and rotation, the sinusoid representing each nanorope has the form

$$
y^{\prime}=y_{0}^{\prime}+A \sin \left(\omega x^{\prime}+\alpha\right) \text {. }
$$

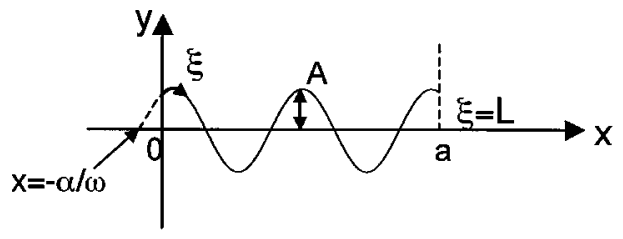

(a)

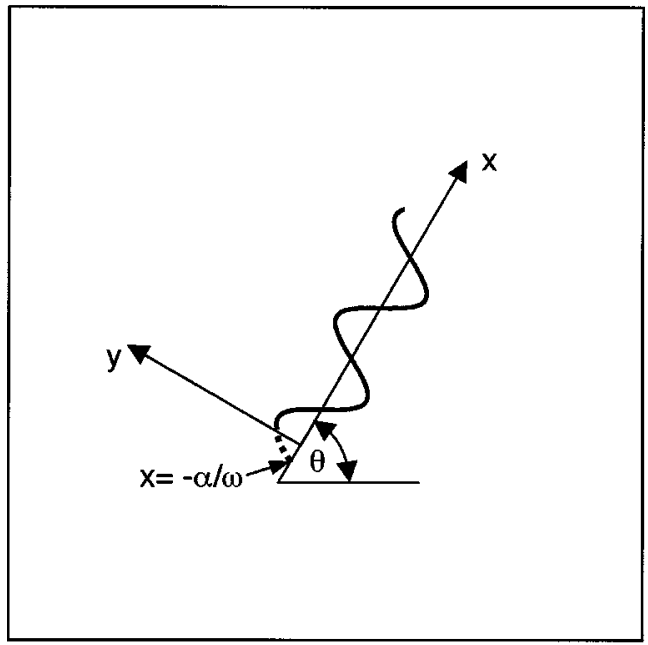

(b)

FIG. 2. Parameters for (a) fiber geometry and (b) network generation.

The four unknown parameters $y_{0}^{\prime}, A, \omega$, and $\alpha$ for each nanorope were determined for each sinusoidal curve using the four randomly selected coordinate points measured $\left[\left(x_{i}^{\prime}, y_{t}^{\prime}\right), i=1,2,3,4\right]$ via simultaneous solution of the following four equations $(i=1,2,3,4)$ :

$$
y_{i}^{\prime}=y_{0}^{\prime}+A \sin \left(\omega x_{i}^{\prime}+\alpha\right)
$$

reduction of these two nonlinear equations via elimination of $y_{0}$ and $A$, as

$$
\frac{\sin \left(\omega x_{1}^{\prime}+\alpha\right)-\sin \left(\omega x_{2}^{\prime}+\alpha\right)}{\sin \left(\omega x_{1}^{\prime}+\alpha\right)-\sin \left(\omega x_{3}^{\prime}+\alpha\right)}=\frac{y_{1}^{\prime}-y_{2}^{\prime}}{y_{1}^{\prime}-y_{3}^{\prime}}
$$

and

$$
\frac{\sin \left(\omega x_{1}^{\prime}+\alpha\right)-\sin \left(\omega x_{2}^{\prime}+\alpha\right)}{\sin \left(\omega x_{1}^{\prime}+\alpha\right)-\sin \left(\omega x_{4}^{\prime}+\alpha\right)}=\frac{y_{1}^{\prime}-y_{2}^{\prime}}{y_{1}^{\prime}-y_{4}^{\prime}},
$$

allowed calculation of $A$ as

$$
A=\frac{y_{1}^{\prime}-y_{2}^{\prime}}{\sin \left(\omega x_{1}^{\prime}+\alpha\right)-\sin \left(\omega x_{2}^{\prime}+\alpha\right)} .
$$

Amplitude $A$ and angular frequency $\omega$ were thus found by simultaneous, numerical solution of Eqs. (5) and (6); we used MATLAB to obtain the values reported here. The period 
TABLE I. Data for sinusoidal ropes in (a) HiPco (annealed) and (b) laserablated (unannealed) NT sheet samples measured from SEM images. For both samples the mean rope diameter was $10 \mathrm{~nm}$. Samples provided by the NanoTech Institute at the University of Texas at Dallas.

\begin{tabular}{|c|c|c|c|}
\hline & period, $T(\mathrm{~nm})$ & amplitude, $A(\mathrm{~nm})$ & $T / A$ \\
\hline \multirow{7}{*}{ (a) } & 396 & 26 & 15.3 \\
\hline & 396 & 35 & 11.3 \\
\hline & 863 & 69 & 12.5 \\
\hline & 1001 & 258 & 3.9 \\
\hline & 2505 & 724 & 3.5 \\
\hline & Mean & & 9.3 \\
\hline & period, $T(\mathrm{~nm})$ & amplitude, $A(\mathrm{~nm})$ & $T / A$ \\
\hline \multirow{5}{*}{ (b) } & 1014 & 84 & 12.0 \\
\hline & 1163 & 158 & 7.4 \\
\hline & 1000 & 49 & 20.3 \\
\hline & 589 & 53 & 11.1 \\
\hline & Mean & & 12.7 \\
\hline
\end{tabular}

$T$ is simply $2 \pi / \omega$. Values of $T$ and $A$ for the ropes sampled are compiled in Table I. Ropes presenting as mildly curved circular arcs were modeled as segments of a sinusoid having large $T / A$.

\section{EFFECT OF FIBER WAVINESS ON NETWORK GEOMETRY}

We term the fiber length between crossings as a fiber segment. We note that we use the term "crossing" to indicate any fiber intersection in the geometry model, and "bond" to indicate a fiber intersection in the micromechanical model; use of the latter term indicates some finite torsional properties assigned to a crossing. The network geometry is specified by the total number of fiber crossings or intersections, for a particular fiber geometry. Sinusoidal fibers can be thought of as infinite, one-dimensional (1D) objects with prescribed $\omega$ (or, alternatively, period $T$ ). Both the mean number of crossings per fiber, and the mean distance between successive intersections along a fiber (i.e., mean segment length) can be calculated from this idealization, given the total number of fiber crossings.

In our simulations, random networks of sinusoidal fibers were generated by depositing $N_{f}$ identical fibers of running length $L$, phase angle $\alpha$, centerline inclination $\theta$, and amplitude $A$, in a unit cell, as shown in Fig. 2(b). End points $x_{i}$ and $y_{i}$, and orientations $\theta_{i}$ were randomly generated, and periodic boundary conditions were imposed on the arrays. To study the effect of increased waviness, period lengths $T$ were systematically decreased, holding all other geometric parameters constant. Figure 3 shows sample random networks of 100 fibers, each comprised of fibers with amplitude $A$ $=0.05$, phase angle $\alpha=0$, running length $L=1$, and $\omega=4 \pi$, $8 \pi, 12 \pi$, and 0 , respectively.

Kallmes and Corte ${ }^{14}$ postulated that the number of crossings $N_{c}$ for random networks of 1D fibers was related to mean fiber length $\bar{\lambda}$ and mean fiber curl $\bar{\tau}$ by

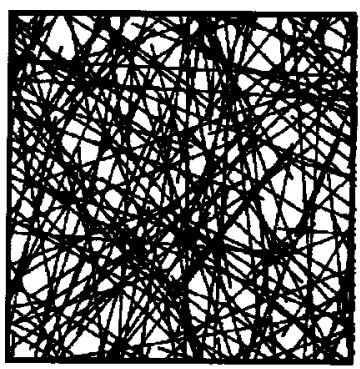

(a)

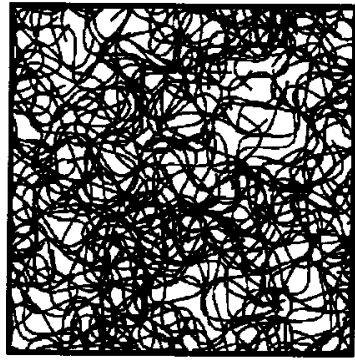

(c)

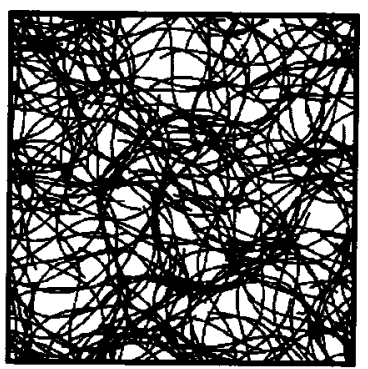

(b)

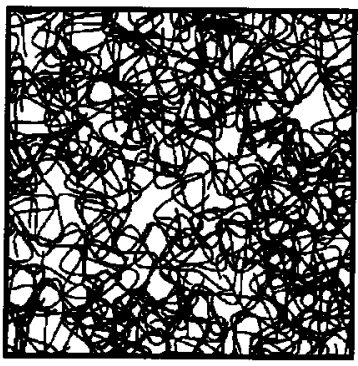

(d)
FIG. 3. Networks of 100 fibers of $L_{f}=1, A=0.05, \alpha=0$, and (a) $\omega=4 \pi$ (b) $\omega=8 \pi(\mathrm{c}) \omega=12 \pi(\mathrm{d}) \omega=0$.

$$
N_{c}=\frac{\left(N_{f} \bar{\lambda}\right)^{2}}{A \pi \bar{\tau}^{2}},
$$

where curl $\tau$ is defined as the ratio of the actual (running) fiber length $\lambda$ to the straight distance between its ends. Our own derivation contradicts this finding, as described in the following paragraphs.

To allow correlation between this model and our own using sinusoidal curves, we first define the straight distance between the fiber ends as $\delta$, where $\lambda$ and $\delta$ are given by

$$
\begin{aligned}
& \lambda=\int_{0}^{a} \sqrt{1+A^{2} \omega^{2} \cos ^{2}(\omega x+\alpha)} d x, \\
& \delta=\sqrt{a^{2}+A^{2}[\sin (\omega a+\alpha)-\sin \alpha]^{2}} .
\end{aligned}
$$

Variable $a$ is the $x$ coordinate of the fiber end point [i.e., value of $x$ for $\xi=L$, as shown in Fig. 2(a)]. The fiber curl is given by $\tau=\lambda / \delta$, or

$$
\tau=\frac{\int_{0}^{a} \sqrt{1+A^{2} \omega^{2} \cos ^{2}(\omega x+\alpha)} d x}{\sqrt{a^{2}+A^{2}[\sin (\omega a+\alpha)-\sin \alpha]^{2}}} .
$$

This relation allows conversion from $\omega$ to $\tau$, given a fiber running length $\lambda$; in the present study, $\lambda$ was taken as unity.

Our expression for the relationship between $\omega$ and number of crossings in a system is briefly described as follows; we provide a more detailed discussion, and also discussion of percolative properties of these arrangements, in another paper. ${ }^{15}$ We initially consider two fibers of length $L_{f}$, arbitrary shape, and random orientation, in an area $A$ [Fig. 4(a)]. Each fiber is divided into $n$ straight segments. The probabil- 


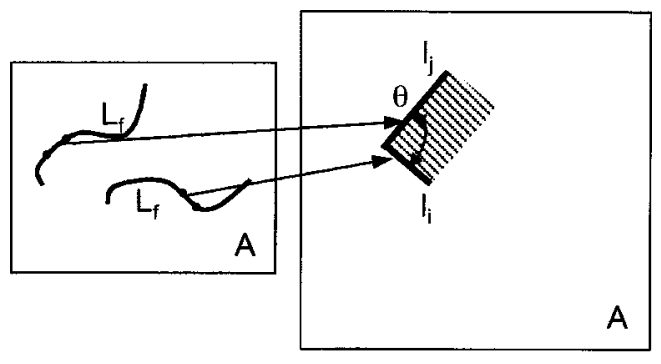

(a)

(b)

FIG. 4. Two wavy fibers of length $L_{f}$ randomly placed in cell of area $A$, shown in (a), and the probability that segments shown intersect, shown as the shaded region divided by total area $A$ in (b).

ity that the $i$ th segment of one fiber will intersect the $j$ th segment of the other is shown as the shaded area divided by the total area $A$ shown in Fig. 4(b), given by

$$
P=\frac{l_{i} l_{j}}{A}|\sin \theta|,
$$

where $l_{i}$ and $l_{j}$ are the segment lengths, and $\theta$ is the angle between the segments. For fibers of random orientation, the probability of intersection can be expressed as

$$
P=\frac{1}{\pi} \int_{0}^{\pi} \frac{l_{i} l_{j}}{A}|\sin \theta| d \theta=\frac{2 l_{i} l_{j}}{\pi A} .
$$

For a network of $N_{f}$ fibers, the total number of fiber segments is then simply the product of $n$ and $N_{f}$. When $N_{f}$ is large, the number of fiber crossings $N_{c}$ can be approximated by

$$
N_{c} \approx \frac{1}{2} \sum_{i=1}^{n x N_{f}} \sum_{j=1}^{n^{*} N_{f}} \frac{2 l_{i} l_{j}}{\pi A}=\frac{L_{\mathrm{total}}^{2}}{\pi A}
$$

where $L_{\text {total }}$ is the total length of fibers in the network. For a network of $N_{f}$ fibers of length $L_{f}$, the number of crossings is thus

$$
N_{c} \approx \frac{\left(N_{f} L_{f}\right)^{2}}{\pi A} .
$$

A plot of the number of fiber crossings versus fiber geometry or waviness is shown in Fig. 5, showing the independence of number of fiber crossings and waviness, and our analytic solution for this parameter.

\section{EFFECT OF FIBER CURVATURE ON RESPONSE}

\section{A. Two-beam model}

We began by modeling two-beam assemblies of curved segments, as shown in Fig. 6. A sinusoidal geometry [Eq. (1)] with phase angle $\alpha=0$ and constant amplitude $A$ $=0.05$, was assumed for the curved segments. Angular frequency $\omega$ was varied to maintain a unit running length in all assemblies (i.e., in all assemblies, $L_{1}=1$, where $L_{1}$ is the running length of $\mathrm{AB}$ and $\mathrm{BC})$. Six cases were considered: $\omega=0$ (i.e., the straight beam case), $\omega=\pi, 2 \pi, 4 \pi, 6 \pi$, and $8 \pi$. For each assembly, $\mathrm{AB}$ and $\mathrm{BC}$ were rigidly connected at B. End A was pinned and end $\mathrm{C}$ was displaced by $X$, in the $x$

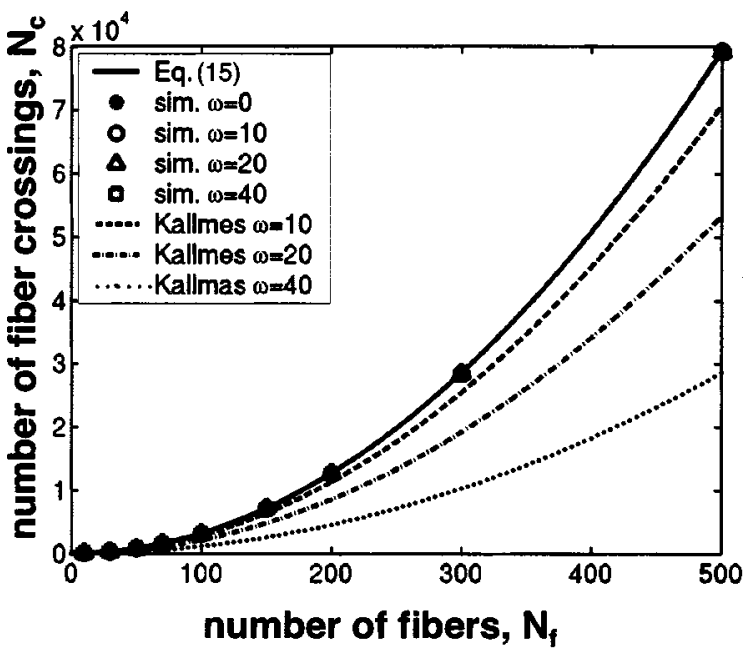

FIG. 5. Predicted number of fibers vs number of fiber crossings per unit area, using our approach and earlier work by Kallmes and Corte (see Ref. 14).

direction. Finite-element analyses were performed on these assemblies to determine the resultant force $Q_{c}$ at $\mathrm{C}$ in the $x$ direction. For each simulation, each curved segment was divided into sixteen elements per period, and each element was modeled as a straight, two-node beam. Analyses were performed for $0 \leqslant \gamma \leqslant \pi$, where $\gamma$ is the intersection angle. In each case, the normalized effective modulus was calculated as

$$
\frac{E_{\text {eff }}}{E}=\frac{Q_{c} L_{2}}{\pi r^{2} X E},
$$

where $L_{2}$ is the distance $\mathrm{AC}$ and $r$ is the beam radius.

Figure 7 shows the results of the two-beam finiteelement analyses. For $\gamma<11 \pi / 12$, the effective modulus of the two-beam assemblies increased with increasing fiber

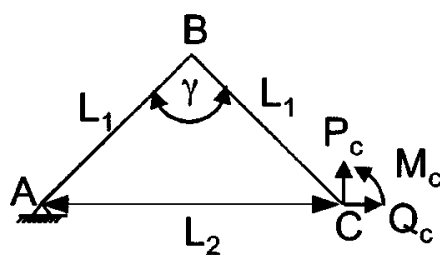

(a)

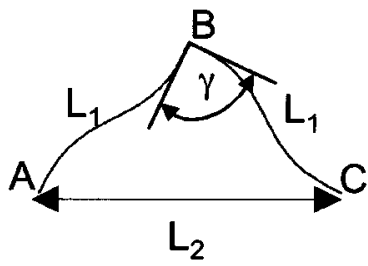

(c)

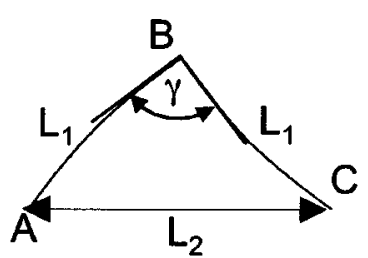

(b)

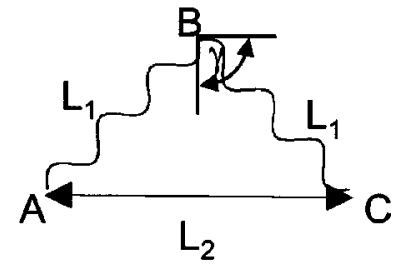

(d)
FIG. 6. Examples of two-beam assemblies used in finite-element analyses, including (a) a straight beam assembly showing reaction forces, and several two-beam constructs, with angular frequencies $\omega$ of (b) $\pi$, (c) $2 \pi$, and (d) $4 \pi$. 


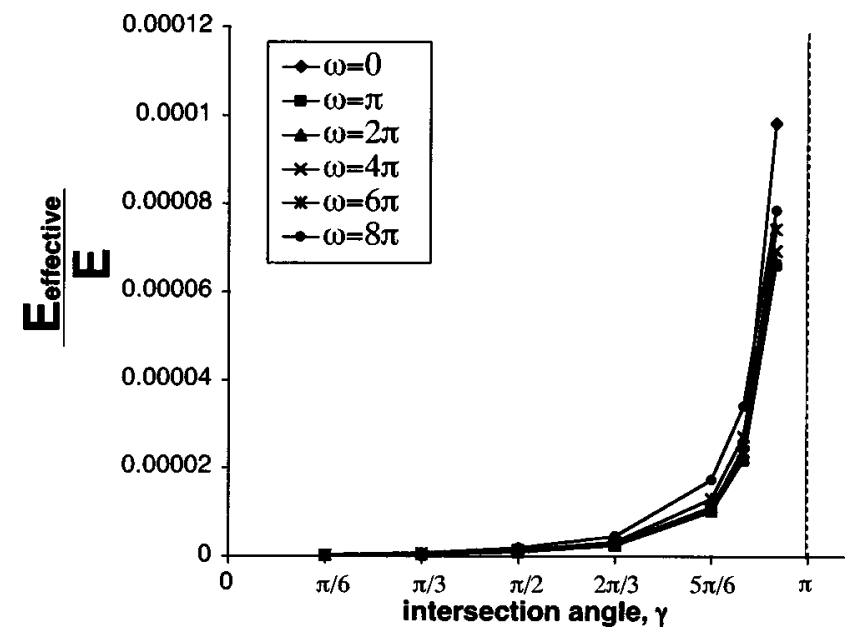

FIG. 7. Normalized $E_{\text {eff }}$ vs intersection angle for two-beam models.

waviness. Beyond this value of $\gamma$, the straight beam assembly was the most rigid assembly. For $\gamma=\pi$, the normalized effective modulus of the straight beam case is, of course, unity, while the corresponding values of effective modulus for the other cases considered range from 0.0002 for $\omega=\pi$ to 0.00014 for $\omega=8 \pi$.

\section{B. Network model}

In earlier work, ${ }^{1}$ we had shown that a random fibrous network of coverage or area fraction $H$ could be adequately modeled using a two-dimensional (2D) network of $N_{f}$ straight beam elements of diameter $d$ randomly distributed in a unit cell with periodic boundary conditions enforced, provided that

$$
N_{f} d=-\log (1-H) .
$$

Briefly, the elastic response of a random fibrous network is largely determined by the total number of fiber crossings, mean number of crossings per fiber, and the mean distance between successive crossing along a fiber, or segment length. ${ }^{14}$ By equating the mean segment aspect ratio of the real and simulated networks via Eq. (17), we are able to model the response of real materials using 2D network simulations. Since the number of fiber crossings (and thus the mean segment length) is independent of fiber waviness, as shown in Fig. 5, Eq. (17) can be applied to networks of wavy fibers as well.

Two-dimensional networks were generated by placement of $N_{f}$ fibers of diameter $d$, unit length, and random end points and orientations, into a unit cell. Periodic boundary conditions were enforced for all networks. For each combination of beam diameter, number of fibers, and curvature, ten networks were generated and analyzed. Each curved beam was divided into 50 straight segments, which were modeled as Euler-Bernoulli beam elements of solid circular cross section. The top boundary of the unit cell was given a displacement $d y$, and the resultant force $F$ on the top boundary was found. The effective modulus $E_{\text {eff }}$ of each network was calculated as

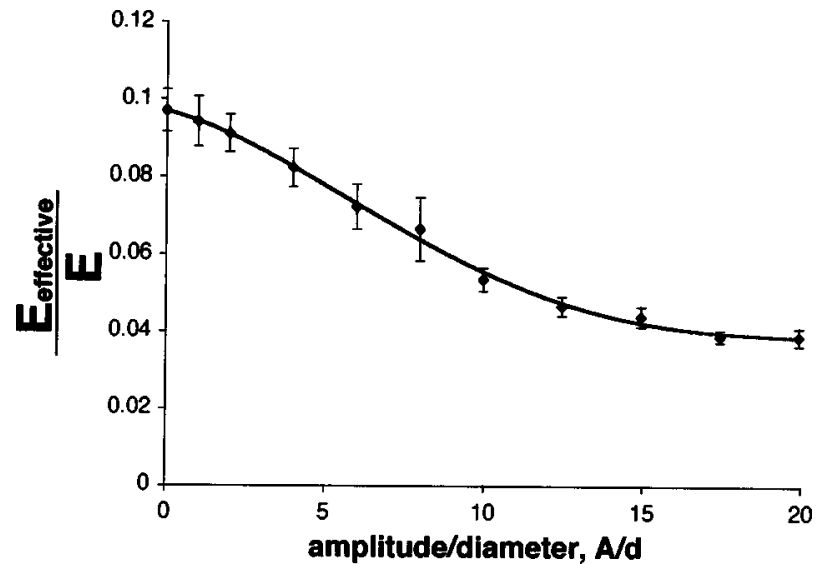

FIG. 8. Normalized $E_{\text {eff }}$ vs $A / d$ showing the effect of reducing fiber waviness via reduction in amplitude. For all simulations, fiber diameter $=0.002$, fiber length $=1$, number of fibers $=200$, and $\omega=10 \pi$.

$$
E_{\mathrm{eff}}=\frac{F}{d y \cdot d} .
$$

Dimensioned values of $E_{\text {eff }}$ were normalized by the fibers' Young's modulus $E$. Four comparisons were made, as described in the following paragraphs. In all the plots of network results (Figs. 8-11), each data point represents the mean normalized $E_{\text {eff }}$ for the ten networks of that specific fiber geometry; error bars represent $\pm 1 \sigma$ for ten realizations.

(1) A comparison of $E_{\text {eff }}$ versus the ratio $A / d$, a quantity determined readily from image analysis of sheets, is shown in Fig. 8. Constant diameter/fiber length ratios $(d / L=0.002)$ and constant angular frequencies $(\omega$ $=10 \pi)$ were used. Fiber amplitude was varied $(0 \leqslant A$ $\leqslant 0.04$ ). Two hundred fibers were used in each simulation, giving networks of area fraction 33\% [per Eq. (17)]. This area fraction is close to the upper bound (32\%) reported for conventional unaligned NT sheets. ${ }^{1,5}$

(2) A comparison of $E_{\text {eff }}$ versus $T / d$, another parameter determined readily from image analysis, is shown in Fig. 9. Constant diameter/fiber length ratios $(d / L=0.002)$, constant fiber amplitude $(A=0.05)$ and variable angular frequencies were used to generate the results. Again, the number of fibers used in each simulation was 200, giving an area fraction $33 \%$ for each network.

(3) A comparison of $E_{\text {eff }}$ versus area fraction is shown in Fig. 10. Constant diameter/fiber length ratios $(d / L$ $=0.002$ ) were used to simulate networks of a range of area fractions from $10 \%-50 \%$, with the number of fibers in each simulation calculated using Eq. (17). Three fiber geometries were considered: straight fibers (case 1), and wavy fibers having constant amplitude/diameter ratios $(A / d=10)$ and period/amplitude ratios of $T / A=10$ (case 2) and $T / A=5$ (case 3).

(4) A comparison of $E_{\text {eff }}$ versus area fraction is shown in Fig. 11. Constant diameter/fiber length ratios $(d / L$ $=0.005$ ) were used to simulate networks of a range of area fractions from $10 \%-50 \%$, with the number of fibers in each simulation calculated using Eq. (17). Three fiber geometries were considered: straight fibers (case 1), and 


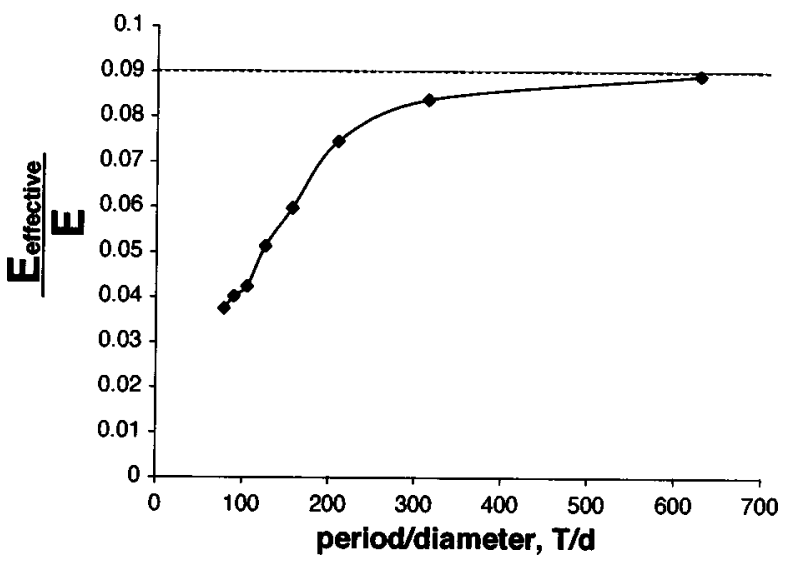

FIG. 9. Normalized $E_{\text {eff }}$ vs $T / d$ showing the effect of reducing fiber waviness via increasing fiber period. For all simulations, fiber diameter $=0.002$, fiber length $=1$, number of fibers $=200, A=0.05$. (Dashed line is the average normalized $E_{\text {eff }}$ for a network of straight fibers of equal diameter.)

wavy fibers having constant amplitude/diameter ratios $(A / d=10)$ and period/amplitude ratios of $T / A=10$ (case 2) and $T / A=5$ (case 3 ).

\section{DISCUSSION}

The first objective of this work was to develop a model for single NT rope curvature encompassing realistic arrangements of NTs in NT mats. Our approach, using a sinusoid to represent fiber segments, allowed reasonable characterization

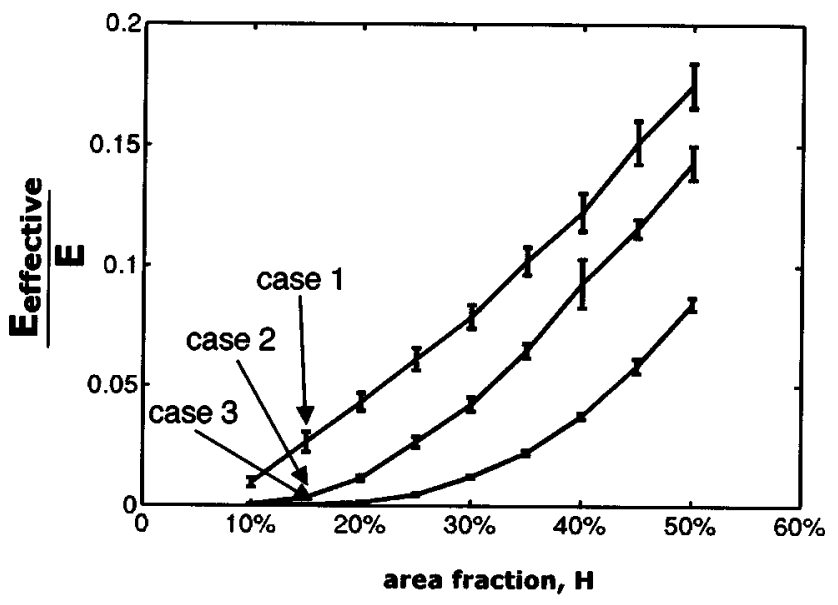

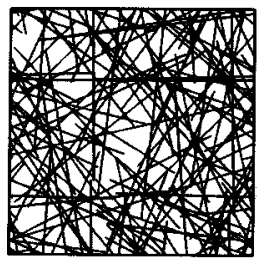

case 1:

$\mathrm{T} / \mathrm{A}=\infty ; \mathrm{A} / \mathrm{d}=10$; $d=0.002$

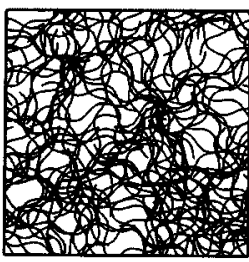

case 2:

$T / A=10 ; A / d=10$; $d=0.002$

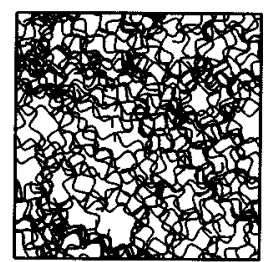

case 3:

$\mathrm{T} / \mathrm{A}=5 ; \mathrm{A} / \mathrm{d}=10$; $d=0.002$
FIG. 10. Normalized $E_{\text {eff }}$ vs area fraction for networks of straight fibers (case 1) and sinusoidal fibers with $A / d=10$ and $T / A=10$ and 5 (cases 2 and 3 , respectively). For all simulations, fiber length $=1$, fiber diameter $=0.002$.

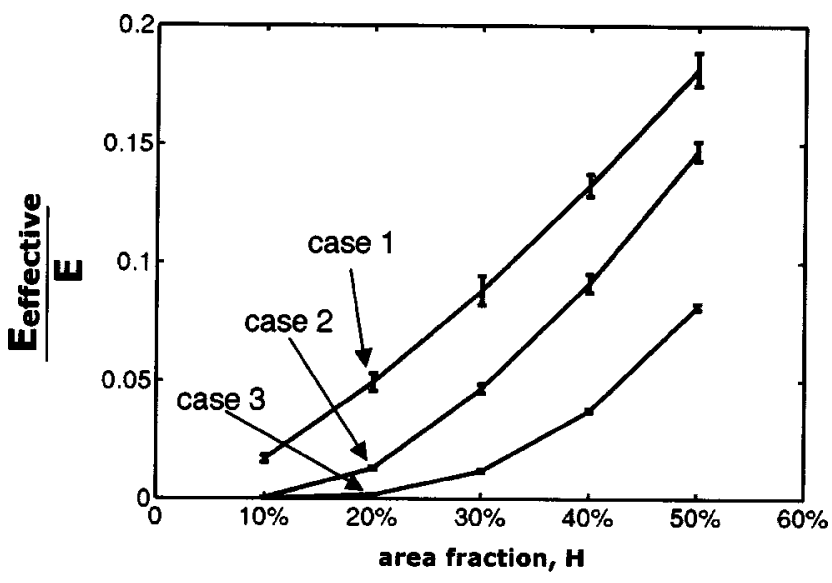

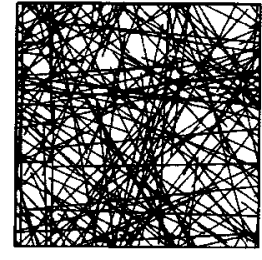

case 1:

$\mathrm{T} / \mathrm{A}=\infty ; \mathrm{A} / \mathrm{d}=10$; $d=0.001$

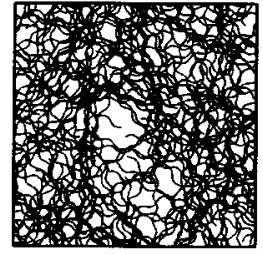

case 2:

$\mathrm{T} / \mathrm{A}=10 ; \mathrm{A} / \mathrm{d}=10$ $\mathrm{d}=0.001$

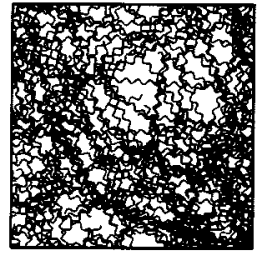

case 3:

$\mathrm{T} / \mathrm{A}=5 ; \mathrm{A} / \mathrm{d}=10$; $\mathrm{d}=0.001$
FIG. 11. Normalized $E_{\text {eff }}$ vs area fraction for networks of straight fibers (case 1) and sinusoidal fibers with $A / d=10$ and $T / A=10$ and 5 (cases 2 and 3 , respectively). For all simulations, fiber length $=1$, fiber diameter $=0.001$.

of the geometries observed, with a few exceptions. For example, some isolated areas of highly curled NTs were visible in several images, forming closed loops on sheet surfaces [Fig. 1(b)]. These looped regions could have been modeled as connected segments of sinusoidal curves having low values of $T / A$ (e.g., 2 or 3). Only ropes that were clearly sinusoidal in shape were measured. Therefore, our reported average values of $T / A$ would certainly lead to lower-thanprobable moduli versus experimental materials, since the majority of the segments in our images appeared to be straight or very mild circular arcs (i.e., reported ratios are smaller than actual average $T / A$ values).

Selecting a single means of fitting a sinusoid to image analysis data proved less trivial than anticipated. In the laserablation-produced sheet, numerical solutions were unrealistic for one curve, because solutions of Eqs. (5) and (6) did not converge for nearly aligned data points (i.e., $y_{1}^{\prime} \approx y_{2}^{\prime} \approx y_{3}^{\prime}$ $\left.\approx y_{4}^{\prime}\right)$. In cases such as this, the nonlinear problem is ill defined, and locations of successive peaks in these fibers must be estimated; amplitudes can be calculated from these. Based on this experience, we recommend in semi-automated image analysis of these systems that data points be collected at locations within single periods, avoiding alignment, in order to minimize the probability of divergence in solution.

Our second objective was to determine the effect of curvature on bond density and other geometric descriptors of NT sheets. In contradiction of classical work by Kallmes and Corte ${ }^{14}$ we found the number of fiber crossings to be inde- 
pendent of fiber waviness, leading to the identification of the number of fiber crossings as a necessary and sufficient parameter to specifying network geometry, for either wavy or straight fibers. The immediate consequence of this is the independence of both the number of crossings per fiber and the mean fiber segment length from fiber waviness.

Our third objective was to determine the effect of curvature on segment response and overall sheet properties. Clearly, the effective network modulus decreases when the fiber waviness is increased, as illustrated by simulations of networks of area fraction $33 \%$ in which we systematically increased fiber amplitude (Fig. 8) or reduced fiber period (Fig. 9). For example, for networks with this area fraction, the mean $E_{\text {eff }}$ of networks of wavy fibers with $A / d=10$ is $55 \%$ that of an average straight fiber $(A / d=0)$ network (Fig. 8). For a network of fibers with $T / d=100$ and area fraction $33 \%$, the mean normalized $E_{\text {eff }}$ is similarly about half that of one comprised of straight fibers (Fig. 9). As described previously, values of $A / d=10$ and $T / d=100$ (i.e., $T / A=10$ ) are physically somewhat higher than actually present in NT sheets, although these values clearly illustrate possible benefits in reduction in waviness. Increases in $E_{\text {eff }}$ can be achieved through fiber straightening via either reducing fiber amplitude (Fig. 8) or increasing fiber period (Fig. 9), relative to fiber diameter. Figure 8 also illustrates that for networks of a given area fraction, the $E_{\text {eff }}$ decreases with increased waviness, but tends to an asymptotic value at high degrees of waviness. Our simulations for $d / L=0.002$ (Fig. 10) and $d / L=0.001$ (Fig. 11) show that response of these networks are within $10 \%$ of each other; thus, our simulation results are not strongly dependent upon diameter, validating use of Eq. (17) in selection of simulation parameters.

The effect of fiber waviness on network response is more significant at lower area fractions. For networks of 50\% area fraction, the mean $E_{\text {eff }}$ for "case 3" networks (comprised of wavy fibers with $T / A=5$ and $A / d=10$ ) was approximately $50 \%$ that of "case 1" networks (comprised of straight fibers) as shown in Figs. 10 and 11. For networks of $10 \%$ area fraction, the mean $E_{\text {eff }}$ for case 3 networks was less than $1 \%$ that of case 1 networks.

In our earlier work, ${ }^{1}$ we developed upper bounds on the effective modulus of NT sheets by considering random networks of straight beam elements rigidly connected at every crossing point. The mean $E_{\text {eff }}$ of random beam networks with every fiber crossing modeled as a torsion spring was found to be within $20 \%$ of that of rigid jointed networks of the same area fraction, even when a very compliant torsion spring of rigidity parameter 0.05 was used $^{1}$ (Fig. 11 in Berhan et al. ${ }^{1}$ ). This suggests that, provided that a physical connection exists at every fiber crossing, the network response is relatively insensitive to the nature of these bonds. Figure 13 in that same work ${ }^{1}$ showed that the theoretical upper bound of $E_{\text {eff }}$ was more than ten times the $E_{\text {eff }}$ of the actual NT sheets, even if the area fractions of the sample materials were overestimated by image analysis of SEM images. These results, together with those presented in Figs. 10 and 11, suggest that the large deviation of the experimental results from the theoretically achievable upper bound can be attributed to two factors: first, a significant discrepancy between fiber crossing

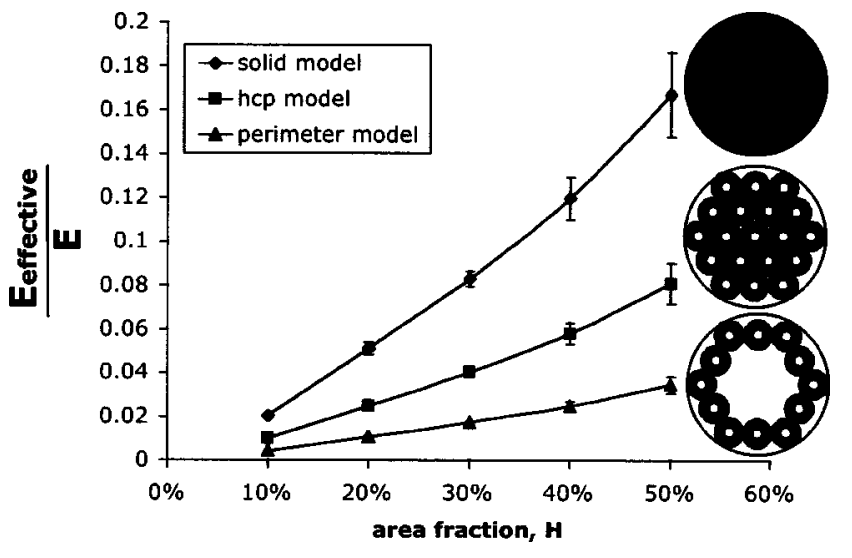

FIG. 12. Normalized $E_{\text {eff }}$ vs area fraction based on model used for cross section of nanoropes (i.e., solid, hcp, and perimeter models).

density (predicted statistically) and fiber bond density, and second, fiber waviness. The relative importance of these factors depends on the actual nanorope waviness (as described by the ratios of $A / d$ and $T / A$ ), and the area fraction of the real material.

Since the network response is largely due to bending for the ranges of area fraction of interest, the results of the solid cross-sectional simulations presented in Figs. 10 or 11 can be applied to the NT sheets by using the moment of inertia of the close-packed rope section to scale the results. An example of this scaling is shown in Fig. 12, which shows the normalized $E_{\text {eff }}$ for NT sheets (modeled as straight segments) using three different cross-section assumptions: a solid circular section, a hcp section with equal load sharing among tubes, and a hcp model assuming only the perimeter ropes are load bearing.

\section{CONCLUSIONS/FUTURE WORK}

Our geometric model allows proper scaling of simulations of finite, 1D fibers, to produce networks with internal characteristics identical to those resulting from infinite 1D fibers. This may be of particular importance in consideration of nanotube mats having no visible fiber ends.

Our mechanics simulations suggest that reduction of waviness in NT ropes may be a plausible route toward improvement of the Young's moduli of random NT sheets, reported in our earlier work. ${ }^{1}$ However, it is of less importance than achieving high bond density; for random sheets, assuring connections among all intersecting ropes ${ }^{16-18}$ appears to be the most direct route toward improving the overall sheet properties. Clearly, there is a discrepancy between statistically predicted bond densities and physical bond densities, based on moduli in these materials. Determining the requirements for a fiber crossing to become a fiber bond is of highest importance. Three-dimensional modeling may be useful in this endeavor. Our work shows that presently, experimental moduli are approximately one-tenth the theoretical moduli of ideal sheets. Use of more advanced image analysis (e.g., confocal Raman spectroscopy ${ }^{19}$ ) in concert with threedimensional simulations, may allow identification of mechanisms for bond formation. Achieving the theoretical moduli 
will also require improvements in connections among individual nanotubes within the ropes, for example, by crosslinking.

Recently, magnetically aligned ribbons have been reported to exhibit effective moduli that are more than ten times those of conventionally manufactured NT sheets. ${ }^{7}$ Further work may be useful in establishing the potential effects of such alignment in NT sheets. This would involve a approach similar to that used in developing the upper bound on Young's moduli for random sheets, possibly using a distribution function to represent selective effects of magnetic alignment.

\section{ACKNOWLEDGMENTS}

The authors gratefully acknowledge support for this work from the DARPA Synthetic Multifunctional Materials Program (Dr. Leo Christodoulou, Program Manager, DARPA-DSO, and Dr. Steve Fishman, Program Manager, ONR), the National Science Foundation, through a PECASE grant (A.M.S.), and W.M. Keck Foundation. We also acknowledge the extremely helpful discussions with, and provision of materials by Prof. Ray Baughman, Dr. Edgar Munoz, and Miles Selvidge of the NanoTech Institute at the University of Texas at Dallas.
${ }^{1}$ L. Berhan, Y. B. Yi, A. M. Sastry, E. Munoz, M. Selvidge, and R. Baughman, J. Appl. Phys. 95, 4335 (2004).

${ }^{2}$ J. N. Coleman, W. J. Blau, A. B. Dalton, E. Munoz, S. Collins, B. G. Kim, J. Razal, M. Selvidge, G. Vieiro, and R. H. Baughman, Appl. Phys. Lett. 82, 1682 (2003).

${ }^{3}$ T. V. Sreekumar, T. Liu, S. Kumar, L. M. Ericson, R. H. Hauge, and R. E. Smalley, Chem. Mater. 15, 175 (2003).

${ }^{4}$ B. Vigolo, A. Penicaud, C. Coulon, C. Sauder, R. Pailler, C. Journet, P. Bernier, and P. Poulin, Science 290, 1331 (2000).

${ }^{5}$ B. W. Smith, Z. Benes, D. E. Luzzi, J. E. Fischer, D. A. Walters, M. J. Casavant, J. Schmidt, and R. E. Smalley, Appl. Phys. Lett. 77, 663 (2000).

${ }^{6}$ P. Poulin, B. Vigolo, and P. Launois, Carbon 40, 1741 (2002).

${ }^{7}$ Y. H. Li, J. Q. Wei, X. F. Zhang, C. L. Xu, D. H. Wu, L. Lu, and B. Q. Wei, Chem. Phys. Lett. 365, 95 (2002).

${ }^{8}$ F. T. Fisher, R. D. Bradshaw, and L. C. Brinson, Appl. Phys. Lett. 80, 4647 (2002).

${ }^{9}$ A. Thess et al., Science 273, 483 (1996).

${ }^{10}$ J. P. Lu, Phys. Rev. Lett. 79, 1297 (1997).

${ }^{11}$ A. G. Rinzler et al., Appl. Phys. A: Mater. Sci. Process. 67, 29 (1998).

${ }^{12}$ J. Liu et al., Science 280, 1253 (1998).

${ }^{13} \mathrm{NIH}$ Image Manual (V1.61) http://rsb.info.nih.gov/nih-image/manual/ contents.html.

${ }^{14}$ O. Kallmes and H. Corte, Tappi J. 43, 737 (1960).

${ }^{15}$ Y. B. Yi, L. Berhan, and A. M. Sastry, J. Appl. Phys. (to be published).

${ }^{16}$ L. Berhan and A. M. Sastry, J. Compos. Mater. 37, 715 (2003).

${ }^{17}$ C. W. Wang, L. Berhan, and A. M. Sastry, J. Eng. Mater. Technol. 122, 450 (2000).

${ }^{18}$ C. W. Wang and A. M. Sastry, J. Eng. Mater. Technol. 122, 460 (2000).

${ }^{19}$ C. Y. Jiang, J. X. Li, J. L. Zhao, U. Kolb, and A. Mews, Nano Lett. 2, 1209 (2002). 